TM-1544

\title{
Flux Creep in a TEVATRON Cable*
}

\author{
M. Kuchnir and A. V. Tollestrup \\ Fermi National Accelerator Laboratory \\ P.O. Box 500, Batavia, Illinois
}

August 22, 1988

*Presented at the 1988 Applied Superconductivity Conference, San Francisco, California, August 21-25, 1988. 


\title{
FLUX CREEP IN A TEVATRON CABLE
}

\author{
M. Kuchnir and A. V. Tollestrup
}

Fermi National Accelerator Laboratory

P.O. Box 500

Batavia, Illinois 60510

\section{Summary}

We have measured the slow magnetization decay of a short sample $(2.3 \mathrm{~cm})$ of Tevatron cable in fields up to $0.3 \mathrm{~T}$. The special susceptometer in development for these measurements is based on a commercial SQUID and is described in detail. The observed decay is logarithmic in time as expected from flux creep in the $\mathrm{NbTi}$ filaments. A strong correlation was found between the decay and the magnetization status of the sample. It is too early yet to present a quantitative correlation between what we observed and the decay observed in the sextupole component of Tevatron dipoles. The detailed understanding of this phenomenon may be instrumental in guiding the search for efficient superconducting synchrotron operational procedures.

\section{Introduction}

In the operation of the Tevatron as a collider the energy is held constant at $150 \mathrm{GeV}$ while proton and antiproton bunches are injected prior to acceleration and storage. Under these conditions the sextupole components of the $\mathbf{7 7 4}$ superconducting dipoles was observed to decay over the course of several hours. The sextupole component of these magnets is partially due to the persistent currents in the microscopic $\mathrm{NbTi}$ filaments of the superconducting cable. We report here measurements of flux creep on a sample of this cable.

\section{Apparatus}

The measuring equipment, schematically shown in Figure 1, consists of a superconducting gradiometer pickup inside the field of a superconducting coil (field coil) whose return flux is channeled through a set of 3 cylindrical $1.6 \mathrm{~mm}$ thick iron laminations. This syotem is shielded by a cylindrical container made out of superconducting $\mathrm{Pb}$ foil which prevents the fluctuations of the room magnetic field from reaching the gradiometer. The whole system is immersed in liquid Helium at ambient pressure. Not shown in the figure is the epoxy-fiberglass (G-11) structure and fiberglass based tape that keeps it rigidly together.

A superconducting $\mathrm{Pb}$ tube shields the superconducting circuit connecting the gradiometer to the SQUID $^{2}$ input. A heater and some thermal insulation at one spot of this tube can keep part of this circuit in the normal state when very large signals are to be generated as when changing the field coil current. This heater, therefore, protects the SQUID from signals that could damage or magnetize it. In this protected condition it measures a highly attenuated time derivative of the flux threading the gradiometer pickup instead of the flux itself as when the protecting heater is off.

The field coil is made with 1500 turns of formvar insulated copper cladded superconducting $0.190 \mathrm{~mm}$ diameter wire over a coil form of $68.0 \mathrm{~mm}$ diameter and $50.8 \mathrm{~mm}$ long. Without the iron laminations it has

*Work supported by the U. S. Department of Energy

Manuscript received August 22, 1988.

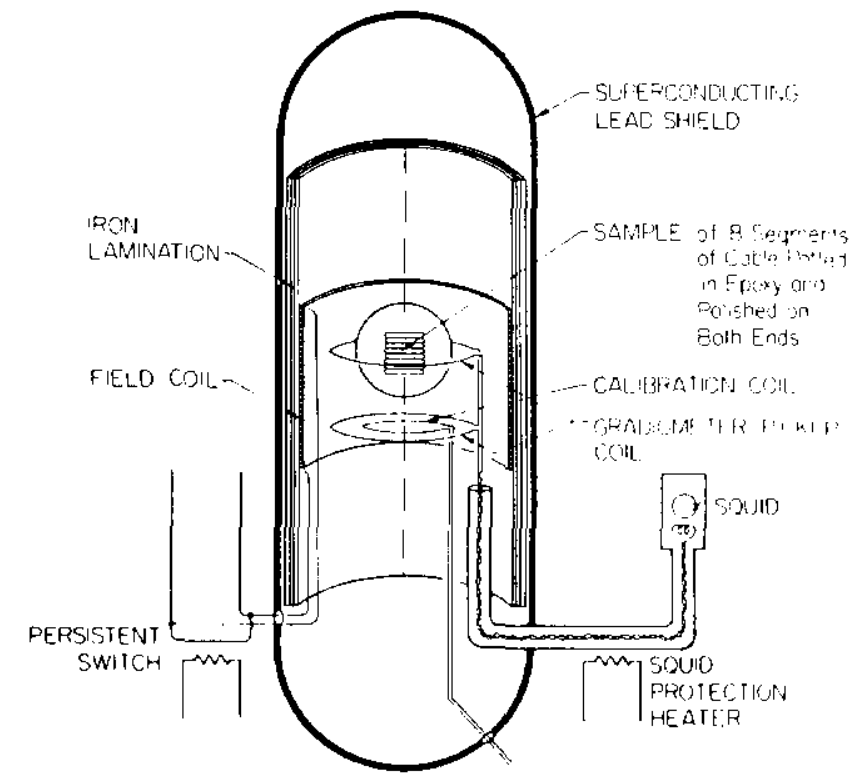

Figure 1. Schematic of measuring apparatus.

an inductance of $0.108 \mathrm{H}$ and an estimated transfer function of $0.03 \mathrm{~T} / \mathrm{A}$.

A persistent current switch shorting the leads of the field coil permits a very stable background field to be maintained for weeks if needed. It also permits the transition from one field value to another to occur without overshoot by the simple process of setting the power supply of the field coil to the desired current and then turning on the persistent switch heater until the field coil current reaches this new value. During these transitions the SQUID input circuit is kept in the protected mode. The time constant of these transitions is of the order of $10 \mathrm{~s}$ under the operating conditions (.20 A, $2.2 \mathrm{~W}$ into the persistent switch heater). Presently the maximum persistent current that this switch can mantain without decay is between 7 and 8 A.

One of the two opposing coils of the gradiometer pickup surrounds the sample and the other a $18.2 \mu \mathrm{H}$ calibration coil of 16 turns and $91.74 \mathrm{~cm}^{2}$ effective area. The identical construction of the opposing coils in the gradiometer permits the calibration of the SQUID output voltage due to the magnetic moment of the sample in terms of the magnetic moment of the calibration coil with a known current (usually a $1 \mathrm{~Hz}$ triangular waveform). Each of the coils of the gradiometer pickup is made with 8 turns of $0.1524 \mathrm{~mm}$ diameter formvar insulated NbTi wire on a $43.48 \mathrm{~mm}$ diameter circle. These coils are $25.9 \mathrm{~mm}$ apart.

The sample is roughly a rectangular parallelepiped volume made out by segments of superconducting cable insulated and pressed as in a magnet and potted in epoxy with both ends polished as for microscopic examination so that it contains no superconducting loops 
due to contacts between filaments in these extremities. The direction of the strands in the samples are perpendicular to the axis of the field coil and gradiometer pickup. The geometry of the above described components is far from properly designed for quantitative measurements. Its purpose was a fast evaluation of the phenomena and difficulties associated with its measurement. Plans for a properly designed apparatus are under way.

The current through the field coil when in the persistent mode is not affected by a typical refill transfer. A typical refill transfer uses 40 liters of He and lasts 3 days. A second SQUID and a cryogenic Hall probe were also used for monitoring the magnetic field away from the gradiometer pickup but no measurements based on them are presented here.

\section{Flux Creep Rate Measurements}

The developmental nature of the apparatus and measuring procedures made it advantageous to have it operated manually. A Nicolet 3091 digital oscilloscope was used instead of a chart recorder for recording the output of the SQUID as a function of time. Software was developed for transferring the contends of this oscilloscope memory via its RS232 port to our VAX cluster, for processing and for graphic presentation using PicSure.

A typical flux creep measurement atarts by protecting the SQUID (powering the heater on its input circuit with .10 A, $0.8 \mathrm{~W}$ ), setting the HP6031A power supply for the field coil to the desired cursent, turning on the persistent switch heater for $60 \mathrm{~s}$, marking the time (to the second) of turning it off and proceeding to turning off the heater protecting the SQUID, marking the time (to the second) of starting the recording by the digital oscilloscope.

In the processing of the data we found that by using $t=0$ as the turning-off time of the persistent switch heater, the accumulated output signal of the SQUID is a straight line function of $\log (t)$. The output of the SQUID ranges from $-10 \mathrm{~V}$ to $+10 \mathrm{~V}$ and is automatically reset to small values upon reaching near those limits. The processing software generates the accumulated output signal for plotting. More than one screenfull ( 4000 points) with different time interval per point can be plotted together. The resulting plots would than have gaps and discontinuities between screenfull traces and perhaps some artifact at the beginning of a trace due to the unlocked status of the tracking software. The relevant feature is the straightness of the line which reflects the flux creep nature $e^{3}$ of the signal and the slope which is a measure of it. One such plot is shown in figure 2 . The calculated slope is also presented as a dashed line to be read against the expanded scale on the right side.

As we accumulated data under different magnetic fields we noticed that the sign and the values of the slopes depended on the history of the magnetic field. The obvious conclusion was that the flux creep was a function of the magnetization of the sample. We therefore proceeded to take the data in some systematic sequence around a hysteresis loop: With the SQUID input in the protected condition and the persistent switch heater on, the field coil current is raised to $10 \mathrm{~A}$ $(0.3 \mathrm{~T})$ and lowered to zero two or three times. The persistent switch heater is turned off and a series of typical flux creep measurements as described above is started. The power supply is turned off during each measurement. The sequence of field coil currents used

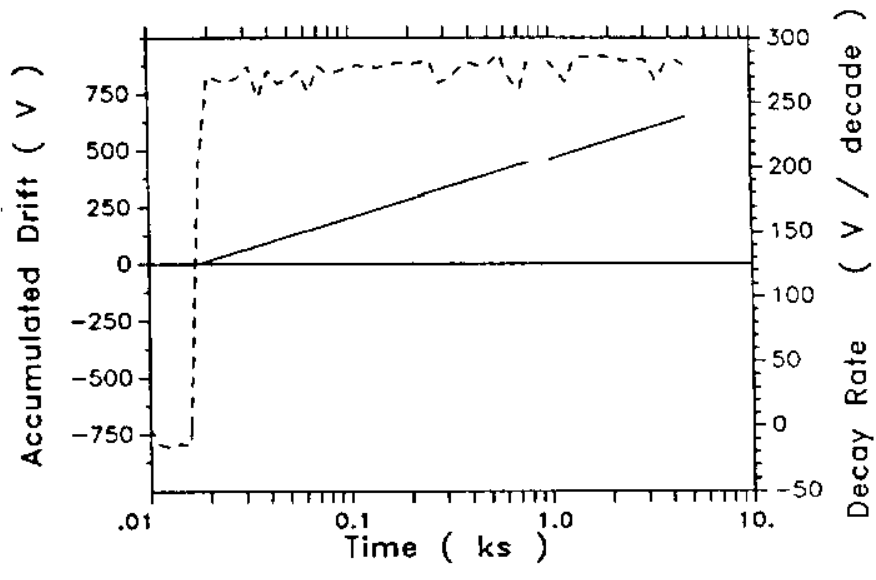

Figure 2. A typical flux creep data point. The solid lines are the processed pickup signal, and the dashed lines their slope.

is monotonically ascending in steps of $1 \mathrm{~A}$. Upon finishing the ascending $7 \mathrm{~A}$ point the current in the coil is raised to $10 \mathrm{~A}$ and the cycle is resumed in descending steps. Figure 3 presents the data collected for a typical Tevatron (zebra) cable and the apparatus background (no sample). The points at $0.30 \mathrm{~T}$ which correspond to $10 \mathrm{~A}$ are not creep rate points, they are included just to indicate the cycle sequence. From this data we see that the magnetic moment signal is essentially due to the sample alone with little contribution from the apparatus.

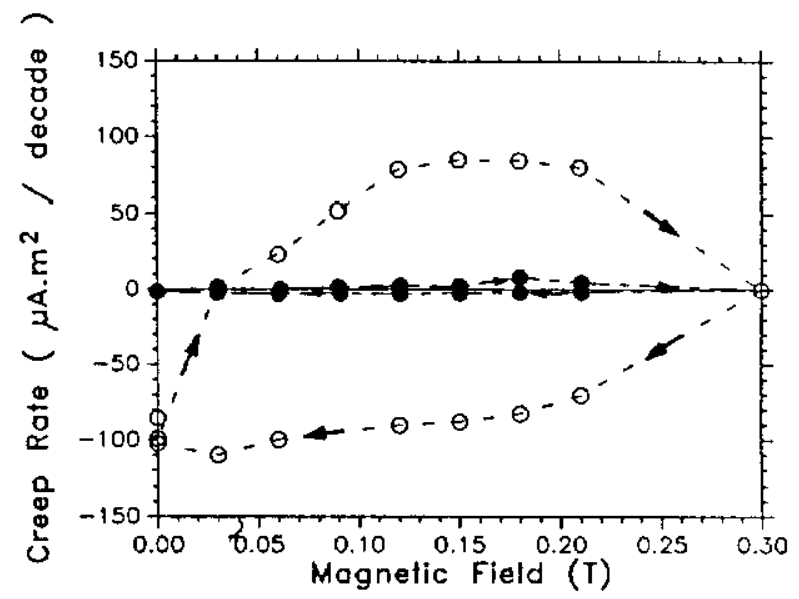

Figure 3. Magnetic Moment creep rate data in a Bystematic sequence of magnetic fields with (open circles) and without (closed circles) a sample of Tevatron cable.

The conversion factor $-.36 \mu \mathrm{A} \cdot \mathrm{m}^{2} / \mathrm{V}$ comes from repeated observations that a $20 \mu A$ triangular waveform through the calibration coil causes as similar triangular waveform (180 $0^{\circ}$ out of phase) of $0.5 \mathrm{~V}_{\mathrm{pp}}$ in the SQUID output.

\section{Magnetization Measurements}

It would be very satisfying to verify independently the appealing indication that the flux creep rate is proportional to the magnetization of the sample. In order to complete this study and have results that can be compared with theory, we are making absolute magnetization measurements in the same apparatus. 
To measure the magnetization of the sample, we calibrate the SQUID output when its input is in the protected mode. Then a $27.4 \mathrm{~mA}_{\mathrm{pp}}, 1 \mathrm{~Hz}$ triangular waveform current through the calibralion coil causes a

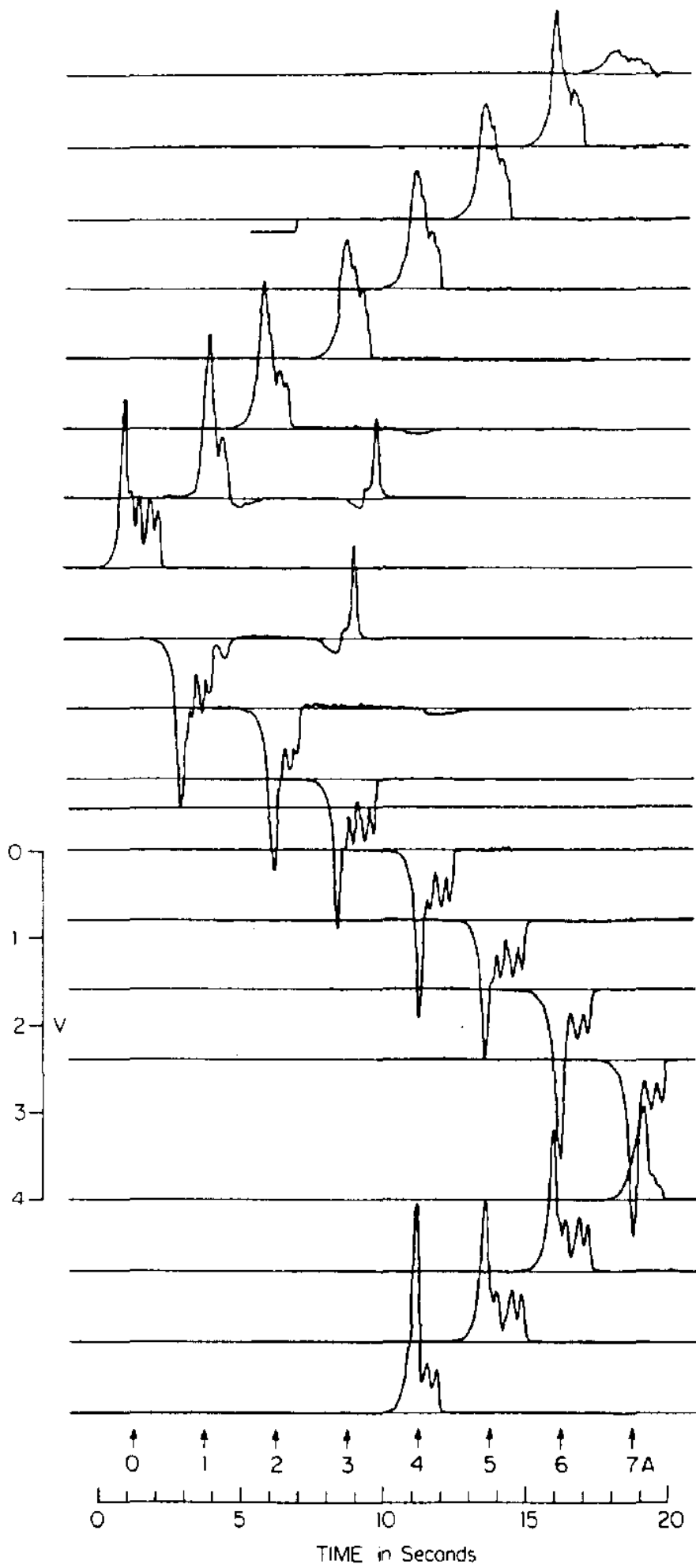

Figure 4. Pulses due to heating the sample above the critical temperature. Two additional pulses are due to the sample cooling back in fields below $H_{c 1}$.

square waveform of $11.46 \mathrm{mV}$ in the SQUID output with transition time constant of $30 \mathrm{~ms}$ and positive slopes corresponding to lower voltages. The calibration factor, therefore, comes out to be $-.09\left(\mathrm{~A} . \mathrm{m}^{2} / \mathrm{s}\right) / \mathrm{V}$. The direct observation of the hysteresis loop proved difficult for instrumental reasons. However, we recently found an elegant way for measuring magnetization in this apparatus: by warming up just the sample above itg critical temperature and recording the change in the magnetic moment detected by the SQUID. This was accomplished by drilling holes in the epoxy near the cables and forcing warm He gas through them for a few seconds. The SQUID input has to be in the protected mode to follow the signal and, therefore, its output has to be integrated with respect to time.

The only sample so far used with this technique is not the Tevatron zebra cable considered above. The 6 cable segments of this sample are rotated $90^{\circ}$ around an axis perpendicular to the gradiometer and field coil axis, so that most of its Nbti filaments have an angle of + or $-7^{\circ}$ with respect to the gradiometer and field coil axis.

Figure 4 presents the SQUID output signal during these flushings (or quenches). One can interpret the structure in the signal as due to lack of synchronism in the quenching of the different cable segments and the extra signal at low field as due to the Meissner Effect. Although the horizontal axis is time, the origin is arbitrary and we manipulated it to reflect the magnetic field in which the flushing was carried out.

Figure 5 presents the areas under the flushing signals obtained along a typical systematic sequence of fields as described above but starting at $7 \mathrm{~A}$ and appropriately converted into $\mathrm{A} \cdot \mathrm{m}^{2}$. We realize that the magnetization status of the sample after a flushing and a field change is not the same as if the flushing did not occur.

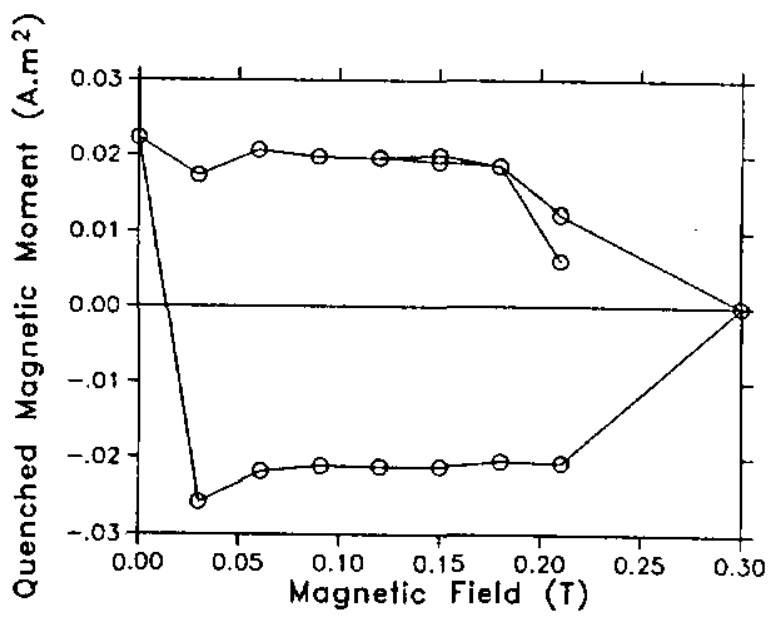

Figure 5. Total magnetic moment change corresponding to pulses in figure 4 .

We are further planning to improve the apparatus and study the effect of magnetic history and temperature on samples of several accelerator magnet cables.

\section{Acknowledgements}

We thank $L$. Theriot for the data transferring software. 
[1] D. A. Finley et al, "Time dependent chromaticity changes in the Tevatron," presented at the IEEE Conference on Particle Accelerators, Washington, D.C., March 16-18, 1987. Also Fermilab publication FN-451, March 1987.

[2] RF SQUID model SHE 30 - Manufactured by BTi, San Diego, CA (619) 453-6300.

[3] M. R. Beasley, R. Labusch and W. W. Webb, "Flux creep in type-II superconductors," Physical Review vol. 181, pp. 682-700, May 1969. 